\title{
Review on Enuresis
}

\author{
Vimal Master Sankar Raj MD \\ Assistant Professor of Clinical Pediatrics, Department of Pediatric Nephrology \\ University of Illinois College of Medicine at Peoria (UICOMP) \\ 420 NE Glen Oak Ave., Suite 401, Peoria, IL 61603, USA \\ vraj@uicomp.uic.edu
}

\begin{abstract}
Abnormalities in micturition pattern can result in a variety of urinary tract symptoms. Incontinence disorders are one such important group of problems clinicians manage in children and adolescents. This literature review goes over the physiology of normal micturition, the neural mechanism involved in the control of micturition, the epidemiology of enuresis, general clinical approach to enuresis, laboratory evaluation, and general principles of management.
\end{abstract}

\section{INTRODUCTION}

Day and night time wetting poses a significant problem for children, parents, and medical practitioners. Though most cases of incontinence are functional, it is important to rule out anatomic, neurologic or other secondary causes of enuresis. Children who have prolonged enuresis have lower self-esteem and feels the continued wetting as a significant life stressor.The following review gives detailed information on the normal physiology of micturition and a step wise approach in diagnosis and management of enuresis.

\section{NORMAL VOIDING}

A normal voiding cycle consists of two phases.

- Filling of the bladder

- Followed by complete emptying of the bladder

Both of these phases are coordinated and active processes. ${ }^{1}$ Bladder filling requires inhibition of bladder contraction along with increased tone of the urinary sphincter complex. As the bladder approaches fullness, tension receptors in the bladder send out afferent signals indicating the need to void. Voluntary voiding then will involve inhibition of urinary sphincter complex and coordinated contraction of the bladder muscles to facilitate complete evacuation of the bladder.

\section{NeUral Control of Micturition}

Regulation of micturition requires extensive connection between several areas in the brain and spinal cord tracts that involve both autonomic and somatic nervous systems. ${ }^{2,3,4}$ Afferent input in regards to bladder sensation of fullness is carried through pelvic and hypogastric nerves. Sensory input from the bladder neck and urethra is carried via the pudendal and hypogastric nerves. These nerves synapse with inter neurons involved in spinal reflex and also with spinal tract neurons that project to the pontine voiding center.

The efferent arm of the micturition cycle involves complex interaction between autonomic and somatic efferent nerves that provide us with voluntary control over the lower urinary tract. Autonomic phase includes both sympathetic and parasympathetic postganglionic nerves. Sympathetic post ganglionic nerves namely the hypogastric nerve release noradrenaline which has an inhibitory action on the detrusor bladder muscle through the $\beta$ receptors. Inversely it exhibits an excitatory action on the bladder neck and urethra through the receptors.

Parasympathetic post ganglionic neurons through the pelvic nerve release both cholinergic (acetylcholine) and non- adrenergic transmitters. Cholinergic stimulation is the major excitatory mechanism in the bladder and acts through the M3 receptors. ${ }^{5}$ Non - cholinergic mode of excitation on the detrusor muscle is mediated through ATP action on the purinergic receptors. Inhibitory input on the urethral smooth muscle is mediated by means of nitric oxide released by the parasympathetic nerves. 
Somatic motor nerves that supplies the striated muscles of the external urethral sphincter arise in the S2-S4 motor neurons and reaches the periphery through pudendal nerves.

\subsection{Neural Circuits in the Control of Continence and Micturition}

During urine storage in the bladder, low level vesical afferent firing occurs. This stimulates the sympathetic outflow in the hypogastric nerve to the bladder outlet and pudendal outflow to the external sphincter. Net result is inhibition of bladder contraction and contraction of external urethral sphincter promoting continence. This represents the guarding reflex for continence and is mediated by the spinal reflex pathway.

In contrast during the elimination phase, intense afferent firing from the bladder stimulates spinobulbalspinal reflex pathway through the pontine micturition center. This stimulates the parasympathetic outflow to the bladder and urethra and inhibits the sympathetic and pudendal outflow. Net result is stimulation of bladder contraction along with relaxation of urinary sphincter complex resulting in complete emptying of the bladder.

\section{Physiology of Micturition}

In infants bladder filling is followed by reflexive emptying. This reflexive micturition cycle is controlled by the sacral spinal cord and occurs almost on an hourly basis. As the bladder capacity increases along with an increase in urinary volume, control of micturition shifts from sacral cord to the pontine voiding center. Establishment of this spinobulbospinal reflex occurs approximately at 6 months of age. ${ }^{6,7}$

Conscious sensation of bladder fullness is reached at around 2 years of age. Voluntary control over bowel and bladder is reached between 2 to 4 years of age with bowel control first before bladder control. Day time bladder control is reached first before nocturnal control. Hence by 4 years of age most children exhibit an adult voiding pattern. But these timing sequences are highly influenced by cultural, economic and family differences. In the western world mean age for urinary continence is around 2 to 3 years of age but can vary between 1 to 5 years. 5 to $10 \%$ of children exhibit episodes of daytime wetting even upto 9 years of age.,

\section{DEFINITIONS}

The standardization committee of the international children's continence society (ICCS) has put forward the following terminology on incontinence

Incontinence - uncontrollable involuntary leakage of urine

Incontinence is either continuous or intermittent.

Continuous incontinence means continuous leakage of urine and occurs exclusively with congenital malformation

Intermittent incontinence is urine leakage in discrete amounts and further classified as daytime and nocturnal incontinence (enuresis). The terms nocturnal incontinence and enuresis are used synonymously.

Nocturnal enuresis is involuntary, intermittent leakage of urine during sleep in a child 5 years or older in the absence of a physical disease regardless of the presence or absence of daytime symptoms. A minimum of one episode a month for at least 3 months is necessary to make a diagnosis.

Definitions for different classification of enuresis are presented in Table 1.

Table1. International Children's Continence Society Terminology

\begin{tabular}{|l|l|}
\hline Enuresis & Involuntary incontinence of urine \\
\hline Enuresis, monosymptomatic & Enuresis without any (other) lower urinary tract symptoms \\
\hline Enuresis, nonmonosymptomatic & Enuresis with (other) lower urinary tract symptoms \\
\hline Enuresis, primary & Enuresis in a child who has previously been dry for less than 6months \\
\hline Enuresis, secondary & Enuresis in a child who has previously been dry for at least 6 months \\
\hline
\end{tabular}

\{Based on Neveus T, von Gontard A, Hoebeke P, et al. The standardization of terminology of lower urinary tract function in children and adolescents: Report from the Standardisation Committee of the International Children's Continence Society. J Urology 2006;176:314-324) 


\section{Review on Enuresis}

\section{EPIDEMIOLOGY}

Nocturnal enuresis affects about 5 million children in the United States. An annual 417000 visits are made to pediatrician's office for incontinence of all etiologies with $38 \%$ for primary nocturnal enuresis. Population based cross sectional studies shows a prevalence rate between 1.5 to $9 \%$ for nocturnal enuresis in children between 6 to 12 years of age. ${ }^{10-14}$ Prevalence rates tend to decrease with age with a reported $20 \%$ in children 5 years of age going down to $1 \%$ by 16 years of age. Every year $15 \%$ of those suffering from bedwetting become dry without treatment.

\section{Pathophysiology}

Pathogenesis of primary nocturnal enuresis (PNE) is unclear but studies done in children have implicated several factors including dysfunction of the bladder, abnormal ant diuretichormone (ADH) production, maturational delay and abnormal sleep patterns.

Urodynamic evaluation done in children with enuresis have shown reduced bladder capacity in many children with PNE and also demonstrated bladder overactivity. ${ }^{15}$ Bladder over activity was present during both day and night. These children were able to manage during wakeful states without any wetting episodes but subsequently have nocturnal incontinence. One other study done by Hagstroem et al has shown abnormal electromyographic activity suggestive of sphincter dyssynergia. ${ }^{16}$

$\mathrm{ADH}$ production normally increases at night decreasing urine production. Rittig and colleagues demonstrated abnormally lower levels of ADH at night in children with enuresis with concomitantly increased urine production. ${ }^{17}$ Some researchers have implicated the lack of response of aquaporin channels to ADH to be one of the factors in enuresis. ${ }^{18}$

Maturational delay in the ability to sense bladder filling and to inhibit bladder contractions at night until the child is awake has been implicated as one of the reasons behind PNE. Functional magnetic resonance imaging (MRI) studies have shown that children with enuresis have delay in maturation of neuronal circuits in prefrontal cortex. ${ }^{19}, 20$ This could explain the fact that enuresis improves with time irrespective of whether treatment is provided or not. Also sleep studies done in enuretic children has shown that they have defective sleep arousal. ${ }^{21-25}$ In these children when urine volume exceeds bladder capacity bed wetting occurs. Underlying emotional cause has been attributed to nocturnal enuresis. ${ }^{26}$ Nocturnal enuresis is also seen more prevalent in children with developmental delay, intellectual disabilities, attention-deficit hyperactivity disorder and autism spectrum disorders.

\section{Evaluation}

A thorough history and physical examination will help the clinician to determine between the different kinds of voiding disorder in children. Differentiating between primary and secondary enuresis is critical for secondary enuresis is more likely to be associated with an identifiable abnormality.

\subsection{History}

An accurate history of incontinence should include onset, pattern, severity and circumstances surrounding the incontinence episodes. An elimination diary looking into specifics of bed wetting, child's urinary habits, drinking habits, bowel movements and presence or absence of urinary tract symptoms could provide important information (Table 2) to differentiate between different causes of enuresis.

The characteristic or urinary stream will also provide clues in the diagnosis. A child with a sensory deficient bladder will usually wet himself/herself completely without any recognition (like sitting in a pool of urine). In contrast a child with urgency-frequency syndrome will tend to squirm, cross the legs, sit on one heal in order to inhibit detrusor contraction. A weak urinary stream is noticed in posterior urethral valve (PUV). Children with sphincter dyssynergia will have staccato urine stream

Any evaluation in a child with incontinence will be incomplete without a thorough evaluation on bowel habits. Constipation tends to occur in about to $10 \%$ of school age children and has been shown to be associated with UTI, urgency-frequency syndrome, incontinence and retention. Dietary habits, frequency of bowel movements, and any history of passing painful hard and blood stained bowel movements needs to be documented. 
Table2. Elimination diary

\section{Questions on bedwetting}

1, Does your child wet himself/herself during day, night or both?

2 , What is the frequency of the wetting episodes?

\section{Questions on voiding habits}

3 , Does your child need to be reminded to urinate?

4, Does he/she urinate before going to bed?

5 , Does he/she urinate after waking up?

6 , Does your child urinate in school?

7, Does your child squirm, cross his/her legs, squat, sit on heels before a urine leak?

8 , Does your child rush to the bathroom to pee and unable to control urination?

9, When your child has a urine leak is he/she not aware of the leak?

\section{Questions on bowel habits}

10, Does your child have a bowel movement every day? If not how often does your child have a bowel movement?

11 , Is there any history of fecal staining on the child's underpants? If yes how often this happens?

12 , Does your child have to push hard for a bowel movement, any history of blood staining on the feaces?

\section{Questions on urinary tract infections}

13, Any past history of urinary tract infection?

14, How many UTI in the past 12 months?

15. What are the associated symptoms with UTI? Any history of fevers, painful micturition, urgency, frquency, foul smelling urine or bedwetting episodes associated with UTI?

16. Was the child on any prophylactic antibiotic when the infection happened? (Break through UTI)

\section{Questions on drinking habits}

17. How much water does your child drink in a day?

18. Does he/she drink any caffeinated drinks?

19. Does he/she drink before going to bed? If so how many cups of water?

\subsection{Physical Examination}

Physical examination should be complete and a thorough evaluation of the abdomen, genitalia, perineum, back and neurological status must be done. Rectal examination may be needed especially in children with c/o needing to strain in order to void and a weak stream. A rare but lethal tumor of the prostate - rhabdomyosarcoma can present with these symptoms in adolescent boys.

Abdominal examination should be thorough with particular attention to identify constipation which can present as lower quadrant fullness. A palpable bladder especially after the child has voided can point towards obstructive etiology including PUV and other neurological conditions.

Genital exam should look into identifying conditions like meatal stenosis in boys, presence of bifid clitoris or other genital abnormalities in girls that can present with voiding disturbances. Continuous pooling of urine in the vaginal vault can present with ectopic insertion of the ureter and should be ruled out in girls with complaints of having never reached dryness.

Inspection of the lower back should be made to look for any signs of spinal dysraphism like tuft of hair, lipoma, hemangioma and sacral dimple. Neurological examination of the lower extremities should include looking for any asymmetrical weakness, loss of strength and abnormal reflexes.

\subsection{Laboratory and Imaging Studies}

Diagnosis of enuresis is made with a complete history and physical examination. No specific laboratory or imaging studies are needed. Depending on specific complaints and concerns for secondary abnormalities further laboratory and imaging studies must be done. Urinalysis with dipstick 


\section{Review on Enuresis}

and microscopic exam along with a urine culture can provide information to rule out urinary tract infection. A negative protein dipstick for glucose, protein with a specific gravity $\geq 1.022$ will rule out polyuric conditions like diabetes mellitus or diabetes insipidus.

Imaging studies again are not needed if clinical suspicion is that of PNE. But if there are concerns for secondary enuresis a renal ultrasound with pre and post void bladder film is warranted. Hydronephrosis in a renal ultrasound may suggest anatomic abnormalities including VUR or PUV. Bladder films especially post void can give information on the functional capacity of the bladder. Further imaging studies including voiding cysto urethrogram (VCUG), radionuclide scans or urodynamic studies may be indicated in specific patients based on the initial evaluation.

A plain radiograph of the abdomen will be helpful in demonstrating constipation especially if the history is not reliable. A plain x-ray can also give information on occult spinal dysraphism, which is a well-recognized cause for secondary enuresis. In the presence of cutaneous manifestations and complaints of enuresis, MRI of the spine may need to be done to screen for occult dysraphism.

\section{TREATMENT}

Primary nocturnal enuresis is a fairly benign condition and most children tend to outgrow it with time. But the condition can be socially stigmatizing and can affect the psychosocial wellbeing of the affected children. In a population based study done in children with enuresis nocturnal enuresis is ranked as the eighth most stressful situation in their life. ${ }^{27}$ Social opportunities can also be limited in children with enuresis like school camps and sleepovers. Children with enuresis suffer from lack of self-esteem and embarrassment, potentially leading to stress and depression. Medical treatment is recommended from 5 years of age particularly if the family and child exhibits a desire to remain dry at night.

Treatment options include both non - pharmacologic and pharmacologic methods. Conservative non pharmacologic methods is encouraged for all children. This includes providing information pamphlet about the condition, giving advice on voiding habits, drinking habits, avoiding caffeine products and encouraging adequate fluid intake. Parents can also try rewarding technique where an agreed behavior like drinking adequately, voiding before sleep and participating in the management after a bed wetting episode are rewarded. Rewarding such behaviors will be more effective than rewarding a dry night which is not under the child's conscious control. Voiding techniques like timed voiding instead of waiting for bladder sensation of fullness can help establish regular voiding habits.Though these conservative measures by themselves may not be effective alone but they can augment the success of other treatment methods. Management of contributing medical conditions like constipation and sleep apnea is of utmost importance and will help in improving the symptoms.

\subsection{Alarm Training}

Conditioning therapy with bell pad alarm is the first line treatment for nocturnal enuresis. Several trials have shown that response to alarm training is more gradual and sustained than any of the pharmacologic methods. Success rate is about $70 \%$ with half of children remaining dry after treatment. ${ }^{28}$ The Bell-Pad devise is based on operant conditioning where urine will complete an electric circuit that triggers an alarm. The patient learns to awaken to the sensations of bladder fullness and micturition.

ICCS guidelines recommend that alarm training be continued for 4 months or until 2 weeks of consecutive dry nights are achieved. Children who did not show any improvement after 6 weeks of training are unlikely to become dry with this method alone. Further behavioral therapy can be used to reinforce alarm training. In a child who has achieved dryness with the alarm technique can be asked to drink more fluids before going to bed while alarm training is continued. This kind of "overlearning" technique can train the child to wake in response to sensation of a full bladder and also reduce relapse after alarm treatment is stopped. "Arousal training" where a child is rewarded for going to the toilet within 3 minutes of an alarm can help in improving motivation and treatment outcomes. ${ }^{29}$

Though bell pad technique can be very effective success rate depends on the motivation of the child and the family. It can be overwhelming and stressful for both the family and child with disruption of sleep pattern. 


\subsection{Pharmacologic Treatment}

\subsubsection{Desmopressin}

Desmopressin (DDAVP) is a synthetic analogue of the anterior pituitary hormone, arginine vasopressin. It has been used in nocturnal enuresis for over 40 years now and works the best for children with polyuria and no functional bladder disturbances. DDAVP acts on the collecting tubules and increases water reabsorption. The intranasal preparation increases the risk for hyponatremic seizures and is no longer indicated in treatment of nocturnal enuresis. Oral preparations are safe and carry much lower risk of electrolyte imbalances when compared to nasal sprays.

DDAVP is given at night about 1 hour before the child goes bed. Starting dose is $0.2 \mathrm{mg}$ and can be increased gradually to a maximum dose of $0.4 \mathrm{mg}$ in 10 to 14 days depending on the response. The drug is well tolerated with minimal side effects. The only potential serious side effect of hyponatremia could be avoided if children restrict drinking (not more than 8 ounces) during night after taking desmopressin. Therapeutic response to desmopressin is quick and over $70 \%$ of treated children will have an improvement in symptoms but relapse rate after discontinuation can be as high as $40 \%$. 30,31 Besides water restriction it will be advisable to hold the medication during an episode of illness, vomiting, diarrhea or poor oral intake with its propensity for electrolyte imbalance. Treatment once started and if responding, it is advisable to continue treatment for 3 months. Then the family and clinician can decide on the continuous usage of medication or to use it only for special occasions like sleepover or school camps. A trial to stop the medication can be tried once a month to see if symptoms have improved without the medication.

\subsubsection{Tricyclic Antidepressants}

Imipramine and other tricyclic antidepressants have been used in the treatment of enuresis with systemic trials showing good therapeutic response when compared to placebo. ${ }^{32}$ Tricyclics by means of its anticholinergic and sympathomimetic action helps in urine retention by its inhibitory action on detrusor muscles. Imipramine is approved for treatment of enuresis in children 6 years and older. The usual starting dose is between 10 to $25 \mathrm{mg}$ at night and the dose can be increased to a maximum of 2 $\mathrm{mg} / \mathrm{kg}$ in 2 weeks' time. Dose should not exceed $50 \mathrm{mg}$ in children 6 to 12 years of ageand not greater than $75 \mathrm{mg}$ in children older than 12 years.

Tricyclics has a vast side effect profile that includes cardiac arrhythmias, hypotension, hepatotoxicity, hematological complications including bone marrow suppression and multiple interaction with other medications. Due to this side effect profile imipramine is not a first line medication for enuresis and should be used only in resistant cases. This medication like DDAVP also has a high relapse rate for symptoms after discontinuation. Since tricyclics can increase vasopressin production in the pituitary, it should not be used in combination therapy with desmopressin.

\subsubsection{Anti-Cholinergic Medications}

Anti-cholinergic medications like oxybutynin exhibits inhibitory action on the bladder muscuranic receptors and hence can stabilize an overactive bladder. This can help increase the bladder capacity. ${ }^{33}$, ${ }^{34}$ Oxybutynin is effective in children who has symptoms of urgency-frequency and may not be effective in primary monosymptomatic nocturnal enuresis. Anticholineric monotherapy is found on trials to be ineffective but can improve treatment response when used in combination with desmopressin, imipramine or enuretic alarms in resistant enuresis. The initial starting dose is $5 \mathrm{mg}$ and can be increasedupto a maximum dose of $15 \mathrm{mg} /$ day.

Side effect profile mainly consist of anticholinergic symptoms namely dry mouth, flushing, constipation, dry eyes and dyspepsia. Oxybutynin if used in combination with imipramine can increase the risk for hyperpyrexia especially in hot conditions. Other newer anticholinergic medications including tolterodine, darifenacin and solifenacin though used in adult population has not yet been approved for use in pediatric age group.

\subsubsection{Psychological Treatment}

ICCS recommends screening for any underlying psychological problem in children with enuresis especially in children with resistant symptoms and co morbid conditions using validated parental questionnaires. ${ }^{35,36}$ If behavioral or emotional conditions are present then further evaluation and treatment should be pursued with referral to a psychologist or psychiatrist. 


\section{Conclusion}

Primary nocturnal enuresis is a fairly benign and common problem encountered in pediatric practice. A thorough history,physical examination aided by laboratory and imaging studies as indicated helps in differentiating primary nocturnal enuresis from other pathological causes of incontinence. PNE carries a wonderful prognosis with a reported $15 \%$ resolution of symptoms spontaneously every year. Several treatment options are available to help with enuresis and hence improve self-esteem and social wellbeing of the affected children. So it is important for the practicing clinician to discuss and offer these treatment options in a timely manner and refer children to specialist care if resistant to therapy.

\section{REFERENCES}

[1] Hjalmas K.Urodynamics in normal infants and children.Scand J Urol Nephrol Suppl1988; 114:20-7.

[2] Morgan CW, de Groat WC, Felkins LA, Zhang SJ. Intracellular injection of neurobiotin or horseradishperoxidase reveals separate types of preganglionic neurons in the sacral parasympathetic nucleus ofthe cat. J Comp Neurol 1993; 331:161-182.

[3] Morgan C, Nadelhaft I, de Groat WC. The distribution of visceral primary afferents from the pelvicnerve to Lissauer's tract and the spinal gray matter and its relationship to the sacral parasympatheticnucleus. J Comp Neurol 1981; 201:415-440.

[4] Steers WD, Ciambotti J, Etzel B, Erdman S, de Groat WC. Alterations in afferent pathways from theurinary bladder of the rat in response to partial urethral obstruction. J Comp Neurol 1991; 310:1-10.

[5] Matsui M, et al. Mice lacking M2 and M3 muscarinic acetylcholine receptors are devoid of cholinergicsmooth muscle contractions but still viable. J Neurosci 2002; 22:10627-10632.

[6] Rushton HG.Wetting and functional voiding disorders.Urol Clin North Am1995 Feb; 22(1):7593.

[7] Stein Z, Susser M.Social factors in the development of sphincter control.Dev Med Child Neurol 1967 Dec;9(6):692-706.

[8] Bloom DA, Seeley WW, Ritchey ML, McGuire EJ.Toilet habits and continence in children: an opportunity sampling in search of normal parameters.J Urol. 1993 May;149(5):1087-90.

[9] Caldwell PH, Deshpande AV, Von Gontard A.Management of nocturnal enuresis.BMJ. 2013 Oct 29; 347:f6259. doi: 10.1136/bmj.f6259.

[10] Maternik M, Krzeminska K, Zurowska A.The management of childhood urinary incontinence.Pediatr Nephrol. 2014 Mar 11 [Epub ahead of print]

[11] Thiedke CC. Nocturnal enuresis. American Family Physician 2003;67:1499-1506.

[12] Forsythe WI, Redmond A. Enuresis and spontaneous cure rate. Archives of Disease in Childhood 1974; 49:259-263.

[13] Glazener CM, Evans JH, Cheuk DK. Complementary and miscellaneous interventions for nocturnal enuresis in children. Cochrane Database Systematic Reviews 2005; April 18;(2): CD005230.

[14] Butler RJ, Heron J. The prevalence of infrequent bedwetting and nocturnal enuresis in childhood. A large British cohort. Scand J Urol Nephrol 2008: 42: 257-64.

[15] Norgaard JP, Hansen JH, Wildschiøtz G, Sørensen S, Rittig S, Djurhuus JC.Sleep cystometries in children with nocturnal enuresis.J Urol. 1989 May;141(5):1156-9.

[16] Hagstroem S, Kamperis K, Rittig S, Rijkhoff NJ, Djurhuus JC.Monosymptomatic nocturnal enuresis is associated with abnormal nocturnal bladder emptying.J Urol. 2004 Jun; 171:2562-6

[17] Rittig S, Knudsen UB, Norgaard JP, Pedersen EB, Djurhuus JC.Abnormal diurnal rhythm of plasma vasopressin and urinary output in patients with enuresis.Am J Physiol. 1989 Apr;256(4 Pt 2):F664-71.

[18] Frokiaer J, Nielsen S.Do aquaporins have a role in nocturnal enuresis? Scand J Urol Nephrol Suppl. 1997; 183:31-2.

[19] Lei D, Ma J, Du X, Shen G, Tian M, Li G. Spontaneous brain activity changes in children with primary monosymptomatic nocturnal enuresis: a restingstate fMRI study. Neurourol Urodynam 2012; 31:99-104. 
[20] Lei D, Ma J, Shen X, Du X, Shen G, Liu W, et al. Changes in the brain microstructure of children with primary monosymptomatic nocturnal enuresis: a diffusion tensor imaging study. PLoS One 2012; 7:e31023.

[21] Norgaard JP, Hansen JH, Nielsen JB, Rittig S, Djurhuus JC.Nocturnal studies in enuretics. A polygraphic study of sleep-EEG and bladder activity.Scand J Urol Nephrol suppl 1989; 125:738.

[22] Koff SA.Why is desmopressin sometimes ineffective at curing bedwetting? Scand J Urol Nephrol Suppl. 1995; 173:103-8.

[23] Boyd MM.The depth of sleep in enuretic school-children and in non-enuretic controls.J Psychosom Res. 1960 Jul; 4:274-81.

[24] Kales A, Kales JD.Sleep disorders. Recent findings in the diagnosis and treatment of disturbed sleep.N Engl J Med. 1974 Feb 28; 290(9):487-99.

[25] Kawauchi A, Imada N, Tanaka Y, Minami M, Watanabe H, Shirakawa S. Changes in the structure of sleep spindles and delta waves on electroencephalography in patients with nocturnal enuresis. Br J Urol 1998; 81(suppl 3):72-5.

[26] Landgraf JM, Abidari J, Cilento BG, Cooper CS, Schulman SL, Ortenberg J. Coping, commitment, and attitude: quantifying the everyday burden of enuresis on children and their families. Pediatrics 2004; 113:334-44.

[27] Butler R, Heron J. An exploration of children's views of bedwetting at 9 years. Child Care Health Dev 2008; 34:65-70.

[28] Glazener CMA, Evans JHC, Peto RE. Alarm interventions for nocturnal enuresis in children. Cochrane Database Syst Rev 2005; 2:CD002911.

[29] Van Londen A, Londen-Barentsen MW, van Son MJ, Mulder GA. Arousal training for children suffering from nocturnal enuresis: a 2 1/2 year follow-up. Behav Res Ther 1993;31:613-5.

[30] Glazener CMA, Evans JHC. Desmopressin for nocturnal enuresis in children. Cochrane Database Syst Rev 2002; 3:CD002112.

[31] Schulman S, Stokes A, Salzman PM: The efficacy and safety of oral desmopressin in children with primary nocturnal enuresis. J Urol 2001; 166: 2427-31.

[32] Glazener CMA, Evans JHC, Peto RE. Tricyclic and related drugs for nocturnal enuresis in children. Cochrane Database Syst Rev 2003;3:CD002117.

[33] Miranda B, Bertraud JE Arano: Efficacy of oxybutynin chloride in children with vesicouretral reflux an detrusor instability. Ann Eur Pediatr 1997; 47(3): 251-257.

[34] Nijman R: Role of antimuscarinics in the treatment of nonneurogenic daytime urinary incontinence in children. Urol 2003; 63: 45-50.

[35] Cortes E, Sahai A, Pontari M, Kelleher C.The psychology of LUTS: ICI-RS 2011.Neurourol Urodyn. 2012 Mar; 31(3):340-3.

[36] Neveus T, von Gontard A, Hoebeke P, et al. The standardization of terminology of lower urinary tract function in children and adolescents: Report from the Standardisation Committee of the International Children's Continence Society. J Urology 2006;176:314-324 